\title{
Use of a portable computed tomography scanner for chest imaging of COVID-19 patients in the urgent care at a tertiary cancer center
}

\author{
David D. B. Bates ${ }^{1}$ (1) * Andriy Vintonyak ${ }^{1} \cdot$ Rennie Mohabir $^{1} \cdot$ Usman Mahmood $^{1} \cdot$ Pat Soto $^{1} \cdot$ Jeffrey S. Groeger $^{2}$. \\ Michelle S. Ginsberg ${ }^{1} \cdot$ Marc J. Gollub ${ }^{1}$
}

Received: 23 April 2020 / Accepted: 25 May 2020 / Published online: 9 June 2020

(C) American Society of Emergency Radiology 2020

\begin{abstract}
To present a novel use of a portable computed tomography (CT) for evaluation of COVID-19 patients presenting to an urgent care center (UCC). Infection control is imperative for hospitals treating patients with COVID-19, even more so in cancer centers, where the majority of the patient population is susceptible to adverse outcomes from the infection. Over the past several weeks, our department has worked to repurpose a portable CT scanner from our surgical colleagues that operates with fixed-parameters to perform non-contrast, helical, thin-slice chest imaging to address the known pulmonary complications of COVID-19. Despite the technical limitations of the portable CT unit that was repurposed for the UCC, diagnostic-quality images in an acute care setting were successfully obtained. Repurposing of a portable CT scanner for use in COVID-19 patients offers a feasible option to obtain diagnostic quality images while minimizing the risk of exposing other patients and hospital staff to an infected patient.
\end{abstract}

Keywords COVID-19 $\cdot$ Portable CT $\cdot$ Pneumonia $\cdot$ Infection control $\cdot$ Coronavirus

\section{Introduction}

Since coronavirus disease 2019 (COVID-19) emerged in December 2019, its rapid spread has overwhelmed healthcare systems, and it was ultimately declared a global pandemic in March 2020 by the World Health Organization [1]. According to the Centers for Disease Control and Prevention (CDC), at the time of this writing, the USA has had 752,003 cases and 35,853 total deaths [2]. New York City [3], an epicenter of the disease, has been particularly hard hit, with data from the NYC Health Department showing 129,788 cases, 34,602 hospitalizations, and 8811 confirmed deaths [3]. The scale of this public health crisis is unprecedented and presents unique challenges for radiology departments and hospitals alike.

The role of diagnostic imaging, namely computed tomography (CT), has been evolving in the evaluation of patients with suspected or confirmed COVID-19. Raptis et al. noted in

David D. B. Bates

batesd@mskcc.org

1 Department of Radiology, Memorial Sloan Kettering Cancer Center, New York, NY 10065, USA

2 Urgent Care Service, Memorial Sloan Kettering Cancer Center, New York, NY 10065, USA a review of current literature that the current evidence as a whole should be considered "low quality," namely due to it consisting of retrospective studies and case series [4]. Additionally, in a commentary to the Lancet by Hope et al., the authors note several challenges to using CT in COVID-19 cases and question its role. Recent guidelines from the Fleischner Society, however, acknowledge several potential scenarios and offer recommendations about when CT may be useful in the assessment of these patients. One of the main issues facing radiology departments is the risk of bringing an infected patient to the CT scanner and potentially exposing other uninfected patients and hospital staff to COVID-19 as it spreads through person to person contact or potentially from contaminated surfaces remains high [5].

To manage infection control, some radiology departments have enacted emergency plans to re-route the workflow that could limit the potential transmission of COVID-19 [6-9]. For example, in our own department when transport of a patient is required from the UCC to the radiology department, the acuity of the patient's condition must be considered with regard to whether the scan can wait until the results of a COVID-19 swab are available. In addition, there are guidelines and recommendations for room disinfection after imaging patients which are evolving frequently, and these also factor into the process of imaging a patient with suspected or confirmed 
COVID-19 in the radiology department. Here, we summarize an initiative at our institution to re-purpose a portable CT scanner from the orthopedic operating room to the UCC for evaluation of COVID-19 patients.

\section{Methods}

The orthopedic and neurosurgery departments at our institution routinely use a portable CT scanner (Airo TruCT, Mobius Imaging LLC, Shirley, MA, USA) in the operating suite. As our department was eager to minimize the risk of disease transmission, elective orthopedic and neurosurgical surgeries were halted due to the COVID-19 pandemic, the scanner was re-purposed for use in the hospital's urgent care center (UCC), which is our institutional equivalent of an emergency department setting. Emergency services do not bring patients to our UCC.

\section{Selection of space and room preparation}

An examination room with the appropriate dimensions within the UCC was selected for the portable CT unit. The room was shielded to reduce radiation exposure to staff and other patients in the UCC with lead-lined walls that complied with local regulations for shielding requirements of $\mathrm{X}$-ray facilities. The portable CT unit has a self-drive system and requires a minimum width of hallways to be $4 \mathrm{ft}$ for safe transport. The unit was brought from the orthopedic operating room to the $\mathrm{UCC}$ and positioned in the prepared room asymmetrically so that a stretcher could be wheeled in alongside it (Fig. 1). Portable suction, oxygen, emergency crash cart, and HEPA filtration device were the other furnishing items in the room as required by the Joint Commission and local regulatory standards.

\section{Technical parameters of portable CT scanner}

The portable CT consists of 32 detectors, $1.06 \mathrm{~mm}$ in size in the longitudinal direction at isocenter (Airo Manual). It has one fixed beamwidth of $33.92 \mathrm{~mm}$, and a fixed reconstruction slice thickness of $1.0 \mathrm{~mm}$, in the same manner, a protocol for non-contrast $\mathrm{CT}$ chest in adult patients was programmed into the scanner with the following parameters: $\mathrm{kV} 120, \mathrm{~mA} 50$, effective $\mathrm{mAs} 67.8$, rotation time 1.92 seconds, slice collimation $1.06 \mathrm{~mm}$, slice width 1.0 $\mathrm{mm}$, feed/rotation $48 \mathrm{~mm}$, pitch factor 1.415 , and CTDI vol $10.1 \mathrm{mGy}$. No intravenous contrast was given, as the unit does not have a built in power injector.

\section{Results}

The first patient imaged with the portable CT scanner was a 48-year-old male with a history of lymphoma, presenting with fever and cough. A nasopharyngeal swab was positive for COVID-19. The patient was scanned inferior to superior with arms above the head. The total scan time was 20.2 seconds, and had a CTDIvol: $10.12 \mathrm{mGy}$, scan DLP of $483.46 \mathrm{mGy}-$ $\mathrm{cm}^{2}$, and a scan length of $42.6 \mathrm{~cm}$.

The non-contrast CT of the chest demonstrated new marked bilateral consolidations and associated ground-glass opacities, predominantly involving the lower lobes and posterior upper lobes (Fig. 2).

\section{Discussion}

Despite the technical limitations of re-purposing a portable CT scanner for use in the UCC, we were able to perform a noncontrast CT chest of diagnostic quality. There was initial concern that due to the fixed scanning parameters, the long exposure time would result in excessive respiratory motion, limiting the quality of the scan. However, the scanner performed adequately, with diagnostic quality images of a patient with confirmed COVID-19 infection.

Despite presenting with symptomatic pulmonary involvement of COVID-19, the patient was able to perform a $20.2 \mathrm{~s}$ breath-hold for the duration of the scan. The decision was made when the protocol was being designed to scan from inferior to superior, to minimize the effect of respiratory motion on the scan, which tends to occur most at the lung bases.

Although it is early in the world's experience with COVID-19, and the role of cross-sectional imaging remains indeterminate, it is important to consider options

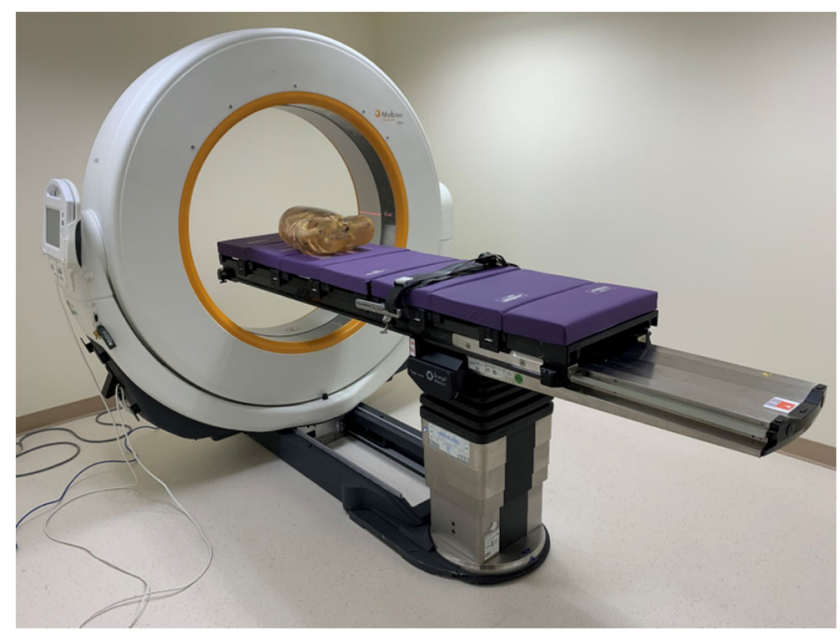

Fig. 1 Image of the portable CT scanner set up in the emergency department at the Memorial Sloan Kettering Cancer Center 


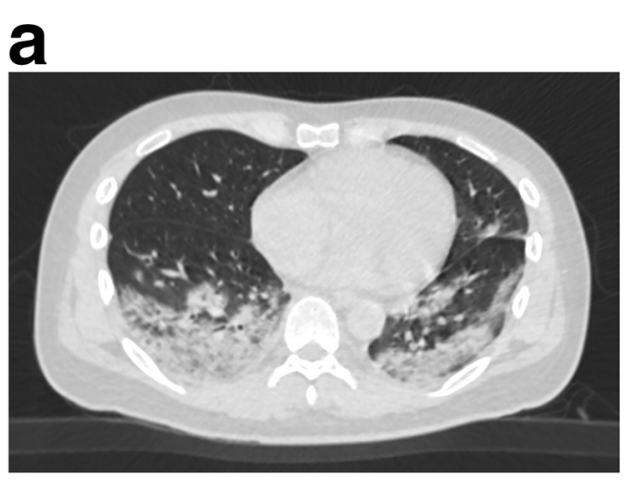

b
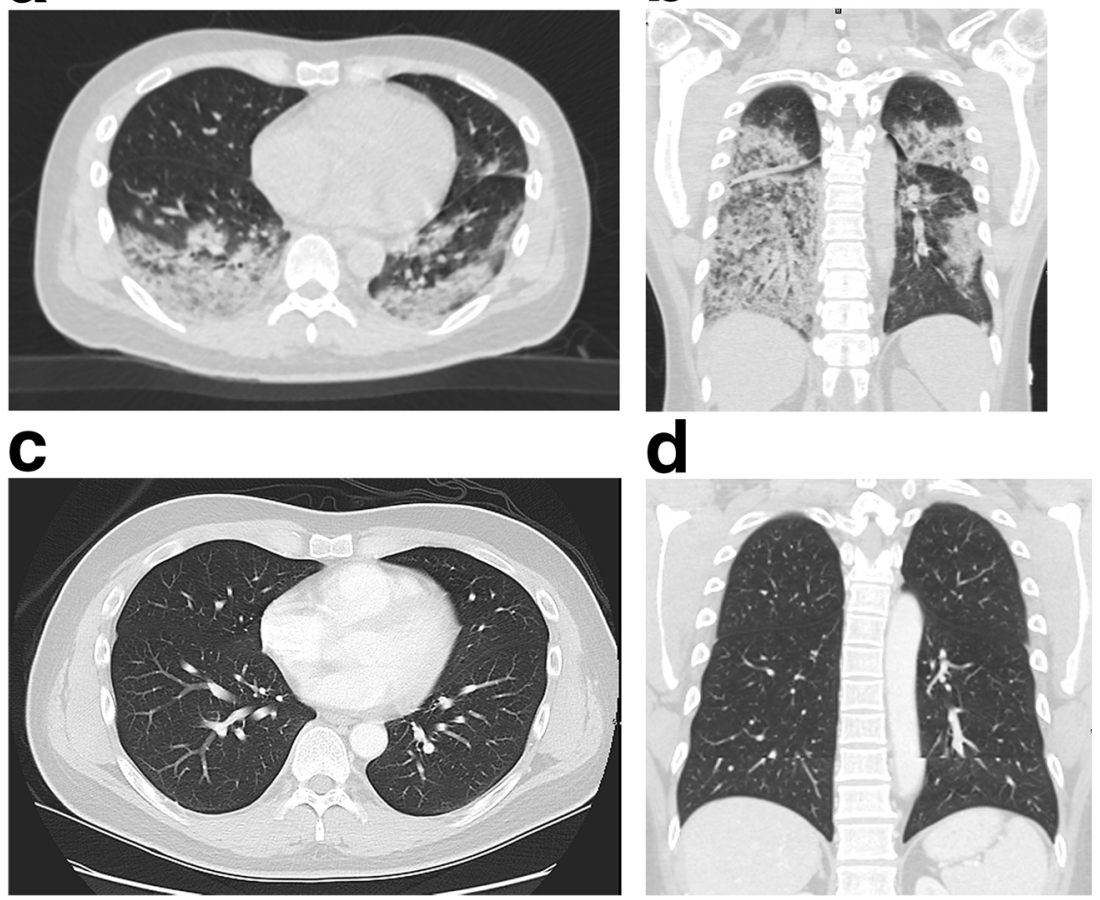

Fig. 2 48-year-old male with non-Hodgkin lymphoma presented with fever and cough. Images acquired on the portable CT scanner (a and b) show bilateral consolidations and ground-glass opacities, consistent with
COVID-19 infection. Images acquired on our departmental conventional CT scanner for the same patient 5 months prior to a routine staging scan are shown for comparison (c and $\mathbf{d}$ ) for obtaining CT of the chest when it is clinically indicated. Some have reported on the role of CT as a prognostic discriminator, with the ability to distinguish between critically ill or severely ill COVID-19 patients and so-called ordinary patients [10]. In addition, the new Fleischner criteria outline certain circumstances in which diagnostic imaging may be warranted, including in patients who have moderate to severe clinical features regardless of COVID-19 status, or for those COVID-19 patients with worsening respiratory status [11]. And although a portable radiograph may be preferable to noncontrast $\mathrm{CT}$, given considerations of preventing transmission of infection, there are likely scenarios in which a non-contrast $\mathrm{CT}$ chest would provide diagnostically useful information. For those situations, a portable CT unit represents another tool in the radiologist's armamentarium that could be considered.

Use of a portable CT scanner has been reported in other situations when there is a clinical reason to do so, for example, to evaluate patients in pediatric [12] or adult intensive care units [13], or to expedite assessment of patients with acute stroke in the emergency department [14]. Although we were able to obtain a diagnostic quality chest $\mathrm{CT}$, it is important to remember our experience has been limited, and we do not know whether this will be the case as our patient cohort grows. One major limitation of this machine is the inability to use a power injector and administer intravenous contrast. The ability to evaluate for a pulmonary embolism, a scenario encountered in this population, is therefore unavailable and would require transport to the radiology department for a CT study. As these patients do present with embolic events and also shortness of breath, this is a clinically relevant limitation of its use.

In summary, we report here the successful implementation of a portable CT unit to perform non-contrast CT chest in an acutely ill COVID-19 patient in the hospital's UCC. Future reports on this technology in this setting will be needed to ensure it is robust and diagnostically acceptable. This may represent a safe method to allow hospitals to image COVID-19 patients for evaluation of lung parenchymal findings while minimizing transport throughout the hospital and therefore the potential spread of infection.

Acknowledgements We would like to acknowledge Jimmy Chin, supervisor of CT at MSKCC, for his work on this initiative.

Funding information The study was supported by the National Cancer Institute (grant no. P30 CA008748).

\section{Compliance with ethical standards}

Conflict of interest The authors declare that they have no relevant conflict of interest related to this work. 


\section{References}

1. (WHO) WHO World Health Organization. https://www.who.int/ $\mathrm{dg} /$ speeches/detail/who-director-general-s-opening-remarks-at-themedia-briefing-on-covid-19\%2D\%2D-11-march-2020. Accessed April 202019

2. Centers for Disease Control and Prevention. https://www.cdc.gov/ coronavirus/2019-ncov/cases-updates/cases-in-us.html. Accessed April 202019

3. Department NH NYC Health Department. https://www1.nyc.gov/ site/doh/covid/covid-19-data.page. Accessed April 172019

4. Raptis CA, Hammer MM, Short RG, Shah A, Bhalla S, Bierhals AJ, Filev PD, Hope MD, Jeudy J, Kligerman SJ, Henry TS (2020) Chest CT and coronavirus disease (COVID-19): a critical review of the literature to date. AJR Am J Roentgenol:1-4. https://doi.org/10. 2214/AJR.20.23202

5. Kooraki S, Hosseiny M, Myers L, Gholamrezanezhad A (2020) Coronavirus (COVID-19) outbreak: what the department of radiology should know. J Am Coll Radiol 17(4):447-451. https://doi.org/ 10.1016/j.jacr.2020.02.008

6. Chandy PE, Nasir MU, Srinivasan S, Klass D, Nicolaou S, Babu SB (2020) Interventional radiology and COVID-19: evidencebased measures to limit transmission. Diagn Interv Radiol 26: 236-240. https://doi.org/10.5152/dir.2020.20166

7. Goh Y, Chua W, Lee JKT, Leng Ang BW, Liang CR, Tan CA, Choong DAW, Hoon HX, Ong MKL, Quek ST (2020) Operational strategies to prevent coronavirus disease 2019 (COVID-19) spread in radiology: experience from a Singapore radiology department after severe acute respiratory syndrome. J Am Coll Radiol. https:// doi.org/10.1016/j.jacr.2020.03.027

8. Huang Z, Zhao S, Li Z, Chen W, Zhao L, Deng L, Song B (2020) The battle against coronavirus disease 2019 (COVID-19): emergency management and infection control in a radiology department. J Am Coll Radiol. https://doi.org/10.1016/j.jacr.2020.03.011
9. Yu J, Ding N, Chen H, Liu XJ, He WJ, Dai WC, Zhou ZG, Lin F, Pu ZH, Li DF, Xu HJ, Wang YL, Zhang HW, Lei Y (2020) Infection control against COVID-19 in departments of radiology. Acad Radiol 27:614-617. https://doi.org/10.1016/j.acra.2020.03.025

10. Li K, Wu J, Wu F, Guo D, Chen L, Fang Z, Li C (2020) The clinical and chest $\mathrm{CT}$ features associated with severe and critical COVID19 pneumonia. Investig Radiol 55:327-331. https://doi.org/10. 1097/RLI.0000000000000672

11. Rubin GD, Ryerson CJ, Haramati LB, Sverzellati N, Kanne JP, Raoof S, Schluger NW, Volpi A, Yim JJ, IBK M, Anderson DJ, Kong C, Altes T, Bush A, Desai SR, Goldin J, Goo JM, Humbert M, Inoue Y, Kauczor HU, Luo F, Mazzone PJ, Prokop M, RemyJardin M, Richeldi L, Schaefer-Prokop CM, Tomiyama N, Wells AU, Leung AN (2020) The role of chest imaging in patient management during the COVID-19 pandemic: a multinational consensus statement from the Fleischner society. Radiology:201365. https://doi.org/10.1148/radiol.2020201365

12. Agrawal S, Hulme SL, Hayward R, Brierley J (2010) A portable CT scanner in the pediatric intensive care unit decreases transferassociated adverse events and staff disruption. Eur J Trauma Emerg Surg 36(4):346-352. https://doi.org/10.1007/s00068-0099127-8

13. Maher MM, Hahn PF, Gervais DA, Seoighe B, Ravenscroft JB, Mueller PR (2004) Portable abdominal CT: analysis of quality and clinical impact in more than 100 consecutive cases. AJR Am J Roentgenol 183(3):663-670. https://doi.org/10.2214/ajr.183.3. 1830663

14. Weinreb DB, Stahl JE (2009) Portable CT imaging of acute stroke patients in the emergency department. Radiol Manage 31(2):41-45

Publisher's note Springer Nature remains neutral with regard to jurisdictional claims in published maps and institutional affiliations. 